\title{
Sommaire du système de surveillance des incidents transfusionnels : 2006-
}

\section{2}

\author{
Mounchili $A^{1 *}$, Leduc $S^{1}$, Archibald $C^{1}$, Miller $\mathrm{J}^{1}$, Hyson $\mathrm{C}^{1}$ \\ ${ }^{1}$ Centre de la lutte contre les maladies transmissibles et les infections, Agence de la santé publique du Canada, Ottawa (Ontario) \\ *Correspondance: aboubakar.mounchili@phac-aspc.gc.ca
}

\section{Résumé}

Contexte : Le Système de surveillance des incidents transfusionnels (SSIT) est un système de surveillance pancanadien établi par l'Agence de la santé publique du Canada (l'Agence) en partenariat avec les provinces et territoires afin de recueillir des données non nominatives sur les réactions indésirables liées à la transfusion observées dans les hôpitaux canadiens offrant des services de transfusion dans le but, essentiellement, d'améliorer la santé des patients.

Objectif : Présenter un résumé des réactions indésirables liées aux transfusions déclarées au SSIT sur la période allant de 2006 à 2012.

Méthodologie : Des hôpitaux de 10 provinces et 2 territoires ont participé au SSIT, recueillant des données sur tous les effets ou incidents liés aux transfusions et les soumettant aux bureaux de coordination du sang des provinces et territoires. Les données ont ensuite été transmises à l'Agence où elles ont été regroupées, épurées, validées et analysées par type de réaction ou résultat. En outre, les taux correspondants ont été calculés en utilisant le nombre total d'unités de composants sanguins transfusées en guise de dénominateur.

Résultats : De 2006 à 2012, un total de 3957 réactions indésirables a été signalé au SSIT, à l'exclusion des réactions allergiques mineures. Sur ce total, 2920 (73,8 \%) étaient liées à la transfusion de composants sanguins et 1036 $(26,2 \%)$ à la transfusion de produits sanguins. Parmi les réactions liées à la transfusion de composants sanguins, les plus courantes ont été : surcharge volémique post-transfusionnelle $(n=1242,42,5 \%)$, réactions allergiques/anaphylactiques/anaphylactoïdes graves $(n=411,14,1 \%)$ et réactions hypotensives $(n=298,10,2 \%)$. Parmi les réactions indésirables liées à la transfusion de produits sanguins, près de la moitié étaient des maux de tête consécutifs à l'administration d'immunoglobuline intraveineuse (IgIV) ( $n=295 ; 28,5 \%$ ) ou des réactions hémolytiques tardives $(n=175 ; 16,9 \%)$. Les décès manifestement imputables à la transfusion ont été extrêmement rares et se limitent à un seul cas atteint d'un syndrome respiratoire aigu post-transfusionnel pour la période allant de 2006 à 2012.

Conclusion : La majorité des réactions attribuables à la transfusion n'a entraîné que des séquelles mineures, voire aucune séquelle. Renforcer le SSIT permettra d'améliorer la surveillance des réactions transfusionnelles indésirables, qui constitue l'une des composantes essentielles de toute stratégie globale de sécurité des patients. Les initiatives actuellement mises en œuvre pour améliorer la qualité des données comprennent l'élaboration d'un algorithme de reconnaissance des cas de surcharge volémique ou de syndrome respiratoire aigu post-transfusionnels ainsi que la recherche de dénominateurs appropriés pour calculer le taux d'incidence des réactions indésirables liées à la transfusion de produits sanguins.

\section{Introduction}

Le Système de surveillance des incidents transfusionnels (SSIT) est un système national de surveillance continue et volontaire établi par l'Agence de la santé publique du Canada (I'Agence) en 2001 dans le but de surveiller les effets indésirables graves et modérés ainsi que certains effets indésirables mineurs liés aux transfusions qui se produisent dans les milieux de soins de santé au Canada. Le SSIT œuvre en collaboration avec les deux fabricants de produits sanguins (la Société canadienne du sang et Héma-Québec) et la Direction des produits de santé commercialisés de 
Santé Canada afin d'effectuer le rapprochement des données recueillies et de garantir la production de rapports complets et exacts.

Le SSIT recueille des données sur les réactions indésirables liées aux transfusions de composants sanguins (globules rouges, granulocytes, plaquettes, plasma et cryoprécipités) et de produits sanguins (dérivés de plasma : albumine, immunoglobuline, facteurs de coagulation, etc.). Les réactions sont signalées par l'entremise d'un important réseau national d'hôpitaux qui couvre toutes les provinces ainsi que deux territoires. Les hôpitaux dans la plupart des provinces et territoires doivent également déclarer les événements indésirables liés à la transfusion aux bureaux de coordination du sang de leur province et territoire, aux fabricants de produits sanguins (Société canadienne du sang et Héma-Québec) et à la Direction des produits de santé commercialisés de Santé Canada. Le programme est administré par le Groupe de travail national sur le SSIT et le Groupe de travail national sur l'examen des données, lequel est constitué de membres provinciaux et territoriaux (principalement les bureaux de coordination des provinces et territoires), ainsi que par des experts dans le domaine de la santé publique, de l'hématologie, des maladies infectieuses et de la médecine transfusionnelle, notamment des travailleurs de la santé de première ligne. Le présent article résume le contenu d'un récent rapport du SSET couvrant la période allant de 2006 à 2012 (1).

\section{Méthodologie}

Les réactions indésirables liées à la transfusion sont définies comme étant des manifestations indésirables et imprévues survenant pendant ou après l'administration de sang, de composants sanguins ou de produits sanguins (dérivés de plasma), qu'elles soient ou non considérées comme liées à l'administration de ces produits. Le SSIT utilise les définitions de cas uniformisées figurant dans le Guide de l'utilisateur du SSIT ainsi qu'un formulaire de collecte de données normalisé rempli par le personnel de surveillance sur le terrain. À noter que la déclaration au SSIT des réactions mineures fréquentes, telles que les réactions transfusionnelles fébriles non hémolytiques, les réactions allergiques mineures et les réactions transfusionnelles sérologiques tardives, n'est pas obligatoire. Les réactions indésirables prises en compte par le SSIT comprenaient les réactions allergiques/anaphylactiques/anaphylactoïdes graves, la surcharge volémique post-transfusionnelle, le syndrome respiratoire aigu post-transfusionnel, les réactions hypotensives, les céphalées liées à l'administration d'immunoglobuline intraveineuse (IgIV) et les réactions hémolytiques aiguës et tardives.

Les cas de réactions indésirables liées à la transfusion ont été examinés et classés en fonction de leur gravité (bénigne, grave et potentiellement mortelle) et de leurs répercussions sur la santé du receveur, soit mineures, pas de séquelles ou décès. Les cas graves étaient définis comme des cas nécessitant une hospitalisation prolongée directement attribuable à l'effet indésirable; des cas entraînant une invalidité ou une incapacité persistante ou importante; des cas nécessitant une intervention médicale ou chirurgicale pour éviter des lésions ou des déficiences fonctionnelles permanentes ou importantes. Les cas étaient jugés potentiellement mortels lorsqu'ils nécessitaient une intervention majeure (vasopresseurs, intubation et transfert en soins intensifs) après la transfusion. Les cas ayant entraîné la mort ont fait l'objet d'investigations approfondies dans les sites concernés visant à déterminer, le cas échéant, la part de responsabilité de la transfusion.

Tous les cas relevés dans les hôpitaux participants étaient compilés et transmis aux bureaux de coordination du sang des provinces et territoires, lesquels étaient chargés d'extraire et de transférer à l'Agence par voie électronique des données non nominatives sur les réactions indésirables graves et modérées liées à la transfusion ainsi que sur certaines réactions mineures, conformément à un accord passé entre les provinces, les territoires et les autorités fédérales. Le transfert des données était effectué tous les trimestres, les données trimestrielles étant transmises à l'Agence dans un délai maximal de six mois. Les données provenant de l'ensemble des provinces et territoires participants ont été examinées, validées et regroupées dans un fichier à des fins d'analyse. Outre les cas de réactions indésirables liées à la transfusion, les provinces et territoires ont également communiqué le nombre d'hôpitaux participant au SSIT pour chaque année de surveillance ainsi que le nombre total d'unités de composants sanguins transfusées. 


\section{Résultats}

De 2006 à 2012, le SSIT a enregistré un total de 3957 effets indésirables liés à la transfusion, dont $2920(73,8 \%)$ liés à la transfusion de composants sanguins. Les réactions liées à la transfusion de composants sanguins les plus courantes ont été la surcharge volémique post-transfusionnelle $(n=1242)$, dont l'incidence s'élevait à 15 cas environ pour 100000 unités de composants sanguins transfusés (figure 1). Parmi les réactions liées à la transfusion de dérivés du plasma, les plus courantes étaient les maux de tête consécutifs à l'administration d'immunoglobuline intraveineuse (IgIV) $(n=295)$ et les réactions hémolytiques tardives $(n=175)$, qui représentaient environ $28,5 \%$ et $16,9 \%$ respectivement.

Sur l'ensemble des réactions, 1835 ont été jugées graves ou potentiellement mortelles (1 505 liées à la transfusion de composants sanguins et 329 liées à la transfusion de produits sanguins). 
Figure 1 : Incidence annuelle des réactions indésirables liées à la transfusion de composants sanguins, SSIT $^{1}$ 2006-2012



${ }^{1}$ SSIT $=$ Système de surveillance des incidents transfusionnels

Parmi les 1693 cas graves ou potentiellement mortels pour lesquels les résultats des patients étaient disponibles, la majorité (1573; 92,9 \%) n'ont entraîné aucune séquelle ou des séquelles mineures, et $79(4,7 \%)$ ont entraîné des séquelles majeures ou à long terme. De 2006 à 2012, 41 décès ont été signalés comme étant sans aucun doute $(n=1)$, probablement $(n=11)$ ou possiblement $(n=29)$ liés à la transfusion, desquels plus de la moitié ont été classés en tant 
que surcharge volémique post-transfusionnelle $(n=13)$ ou syndrome respiratoire aigu post-transfusionnel possible $(n=12)$.

Les réactions indésirables potentiellement mortelles représentaient $7 \%(n=270)$ des réactions indésirables signalées sur la période allant de 2006 à 2012, la majorité de ces cas (92,2\%) ayant découlé de la transfusion de composants sanguins. Les réactions indésirables liées à la transfusion les plus couramment signalées ont été la surcharge volémique post-transfusionnelle $(n=94)$ et les réactions allergiques/anaphylactiques/anaphylactoïdes graves $(n=39)($ figure 2$)$.

Figure 2 : Réactions indésirables potentiellement mortelles liées à la transfusion, SSIT¹ 2006-2012



${ }^{1}$ SSIT $=$ Système de surveillance des incidents transfusionnels

Dans la grande majorité des cas (96\%), les réactions indésirables liées à la transfusion recueillies par le SSIT de 2006 à 2012 ont eu des répercussions mineures ou n'ont entraîné aucune séquelle. Un total de 41 décès liés à la transfusion sanguine a été signalé entre 2006 et 2012; toutefois, la responsabilité de la transfusion n'a été définitivement retenue que pour un seul cas qui avait contracté un syndrome respiratoire aigu post-transfusionnel. Dans tous les autres cas, la relation entre le décès et la transfusion a été jugée tout au plus probable $(n=12)$ ou possible $(n=28)$. Le nombre d'unités de composants sanguins transfusées annuellement dépassant le million, le taux de létalité correspond à environ 5 cas pour un million d'unités (figure 3 ). 
Figure 3 : Réactions indésirables graves liées à la transfusion, SSIT¹ 2006-2012

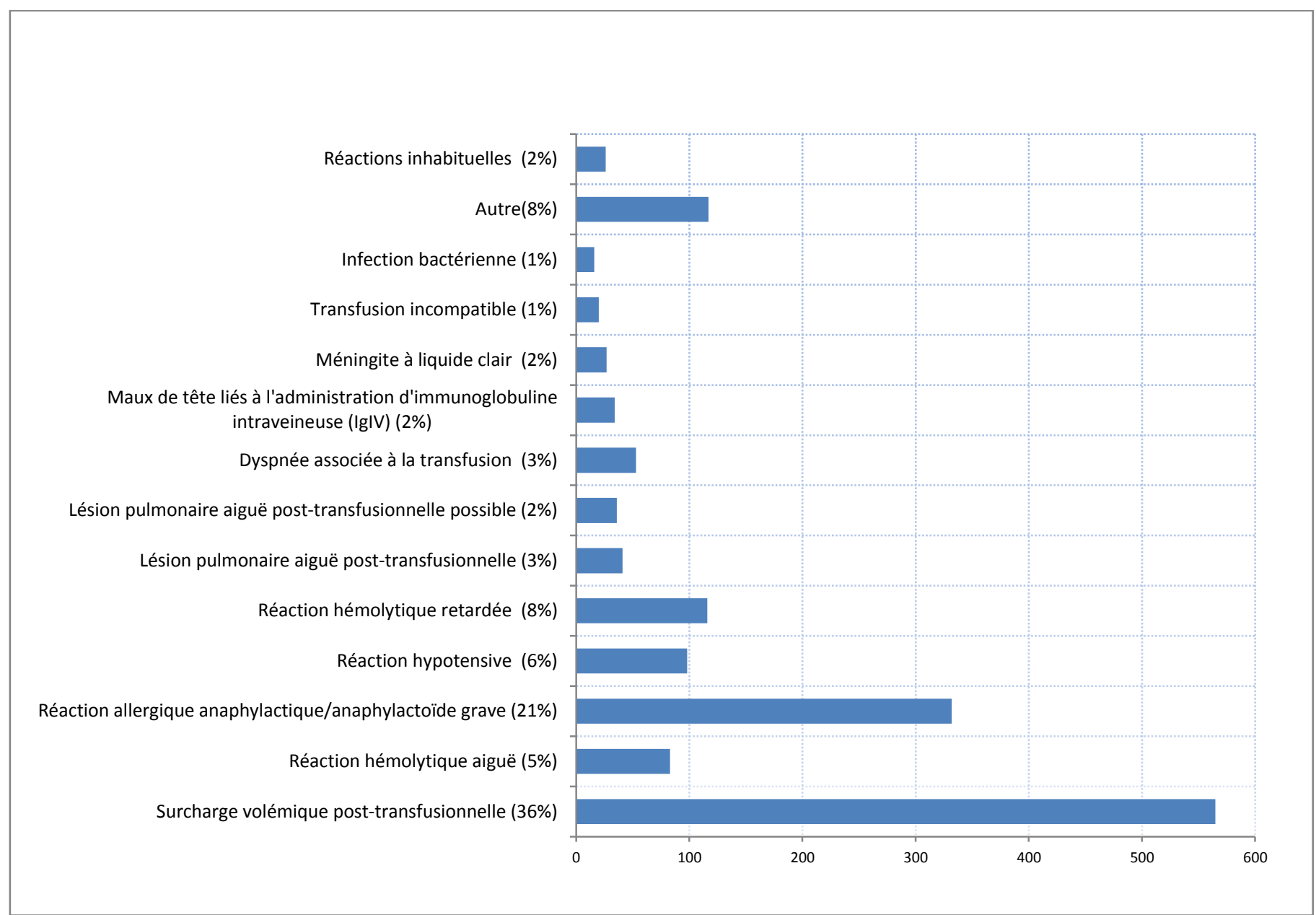

${ }^{1}$ SSIT $=$ Système de surveillance des incidents transfusionnels

\section{Conclusion}

Le SSIT est un système véritablement pancanadien qui permet de recueillir l'ensemble des réactions indésirables liées à la transfusion survenant dans les hôpitaux du Canada. Plusieurs initiatives ont été mises en œuvre par le Groupe de travail national sur le SSIT dirigé par l'Agence dans le but d'améliorer non seulement le système de déclaration, mais également la qualité des données déclarées. Parmi ces initiatives, retenons l'élaboration d'un algorithme qui permette de différencier les cas de surcharge volémique et les cas de syndrome respiratoire aigu liés à la transfusion et la recherche d'une méthode de normalisation des données utilisées en guide de dénominateur pour les produits sanguins. Le maintien du partenariat établi entre l'Agence, les fabricants de produits sanguins (Société canadienne du sang et Héma-Québec), les provinces et territoires et Santé Canada est essentiel pour assurer la déclaration en temps opportun de données de surveillance exactes qui permettent d'améliorer les politiques et procédures relatives à la sécurité des transfusions et ultérieurement de renforcer la sécurité des patients dans tous les hôpitaux canadiens.

\section{Remerciements}

Remerciements : L'élaboration du Système de surveillance des incidents transfusionnels (SSIT) n'aurait pas été possible sans les efforts de collaboration et l'engagement continu de bureaux de coordination du sang provinciaux et territoriaux, du personnel de la médecine transfusionnelle des hôpitaux participants, de la Société canadienne du sang et de Héma- 
Québec. L'engagement de ces groupes à réduire les réactions et incidents indésirables liés aux transfusions et à améliorer la sécurité des patients a mené à la collecte et à l'analyse des données du SSIT pour la période 2006-2012.

\section{Conflit d'intérêts}

Aucun

\section{Financement}

Ce travail a été appuyé par l'Agence de la santé publique du Canada.

\section{Référence}

(1) Agence de la santé publique du Canada. Le Système de surveillance des incidents transfusionnels (SSIT): Résultats sommaires de 2006 à 2012. Ottawa: Centre de la lutte contre les maladies transmissibles et les infections, ASPC; 2014.

http://www.phac-aspc.gc.ca/hcai-iamss/ttiss-ssit/index-fr.php 